Supplement of Hydrol. Earth Syst. Sci., 25, 1365-1388, 2021

https://doi.org/10.5194/hess-25-1365-2021-supplement

(c) Author(s) 2021. CC BY 4.0 License.

(c) (i)

Supplement of

\title{
Coordination and control - limits in standard representations of multi-reservoir operations in hydrological modeling
}

Charles Rougé et al.

Correspondence to: Charles Rougé (c.rouge@ sheffield.ac.uk)

The copyright of individual parts of the supplement might differ from the article licence. 


\title{
Supplement
}

\begin{abstract}
This supplementary information contains 1) results for the offline model devised during the drought event (Section 4.3 of the main paper), and 2) figures comparing the simulated ensemble with the historical record, for storage, inflows and outflows of the five reservoirs on the main branch of the Upper Snake River Basin (USRB; see Table 2 in the main paper)
\end{abstract}

\section{Offline model results}

5 Figures 1 and 2 correspond to the modeling experiment described in the last paragraph of Section 4.3 of the main paper. It described a policy modification in the simulation, at Palisades in 2012

\section{Comparison of simulations with the historical record}

Figures 3 to 7 read the same way as Figure 5 in the main paper; we refer the reader to Section 4.1.1 for detailed explanations on how the figures are constructed. All figures represent the full eight-year simulation period, 2009-2016.

\section{Acknowledgments}

This work was supported by the U.S. Department of Energy, Office of Science, Biological and Environmental Research Program, Earth and Environmental Systems Modeling, MultiSector Dynamics, Contract No. DE-SC0016162 and the material based upon work supported by the National Science Foundation under Grant No. 1639524 in the Innovations at the Nexus of Food, Energy and Water Systems (INFEWS) program. 



Figure S1. Results from the coordinated offline reservoir water balance model (dotted blue lines), compared with hydrological model sim-ulation results from non-coordinated operations (continuous black lines): min, max values and quartiles for both ensembles. Figures show June-November 2012. Differences in storage (panels (a) and (b) for Palisades and American Falls respectively) are due to extra releases from the former reservoir during the summer season. 


\section{a) Jackson Lake}

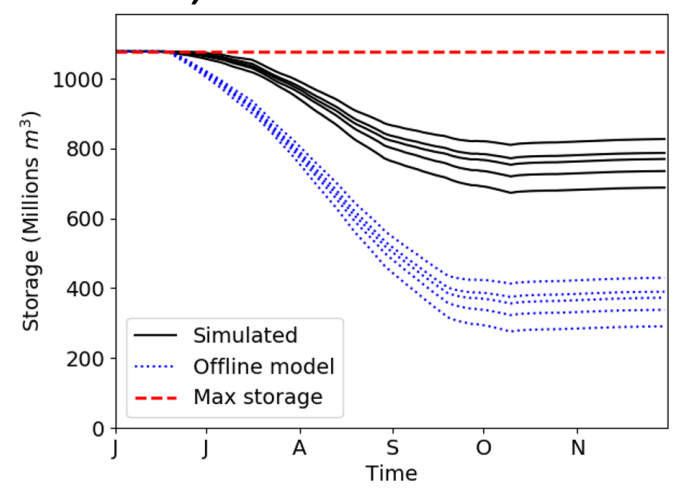

b) Palisades

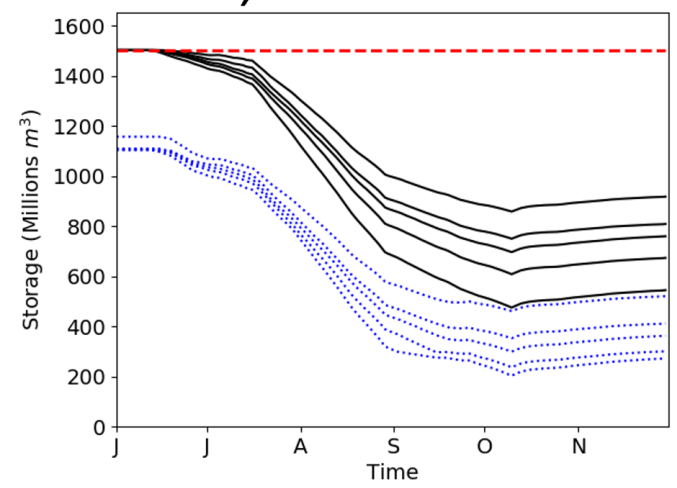

c) American Falls



Figure S2. Results from the coordinated offline reservoir water balance model (dotted blue lines), compared with hydrological model sim-ulation results from non-coordinated operations (continuous black lines): min, max values and quartiles for both ensembles. Figures show June-November 2013. Differences in storage (panels (a), (b) and (c) for Jackson Lake, Palisades and American Falls respectively) are due to extra releases from Jackson Lake and Palisades during the summer of 2013, and from consequences of the extra release from Palisades towards American Falls the summer before. 


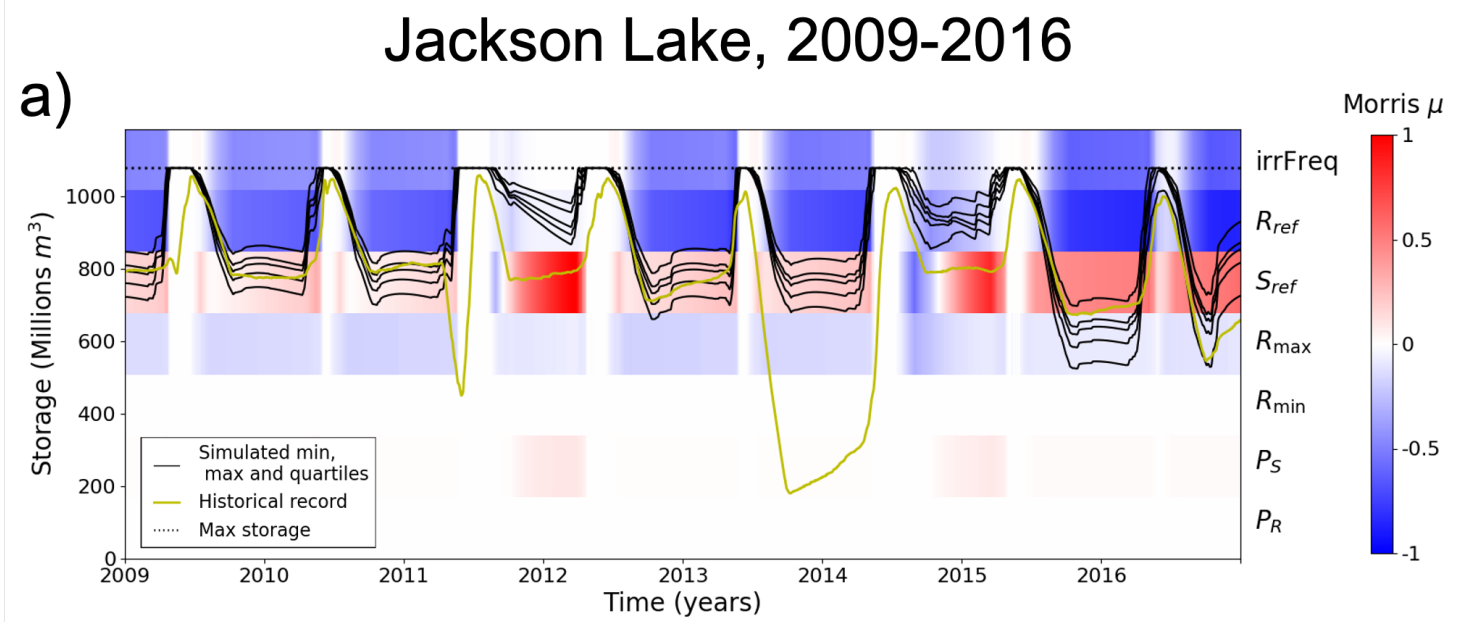

b)

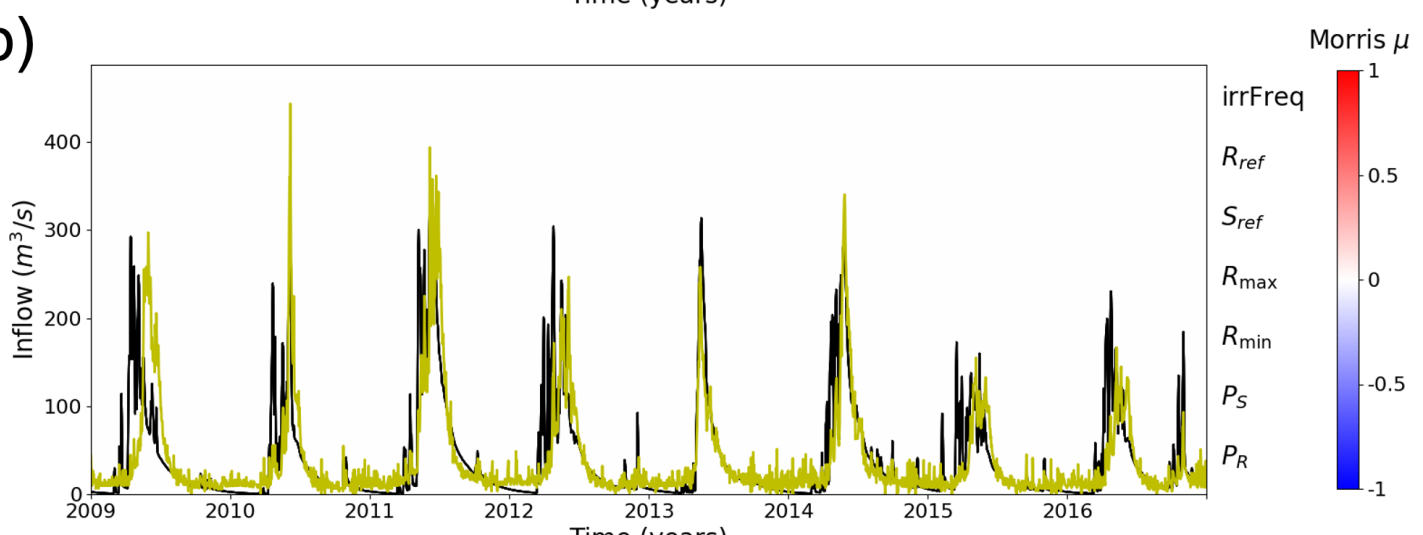

c)

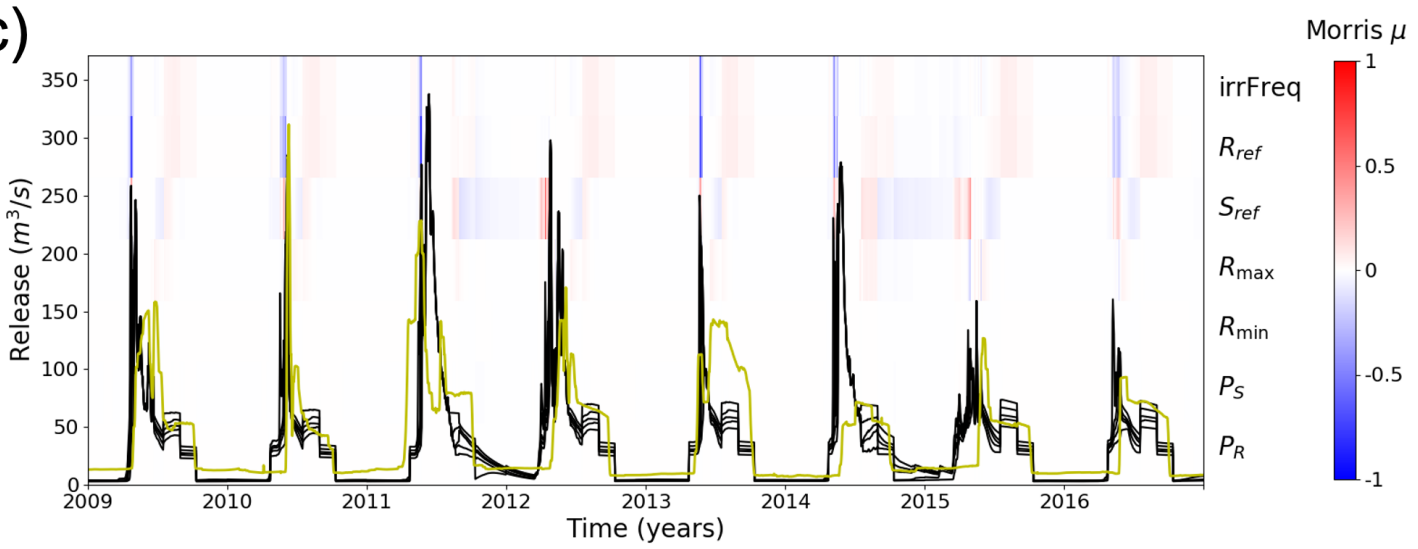

Figure S3. Simulated values (max, min and quartiles, shown with black lines) with historical values (gold line), and sensitivity to input variables (background), for (top to bottom): Jackson Lake inflow, release and storage. 


\section{Palisades, 2009-2016}
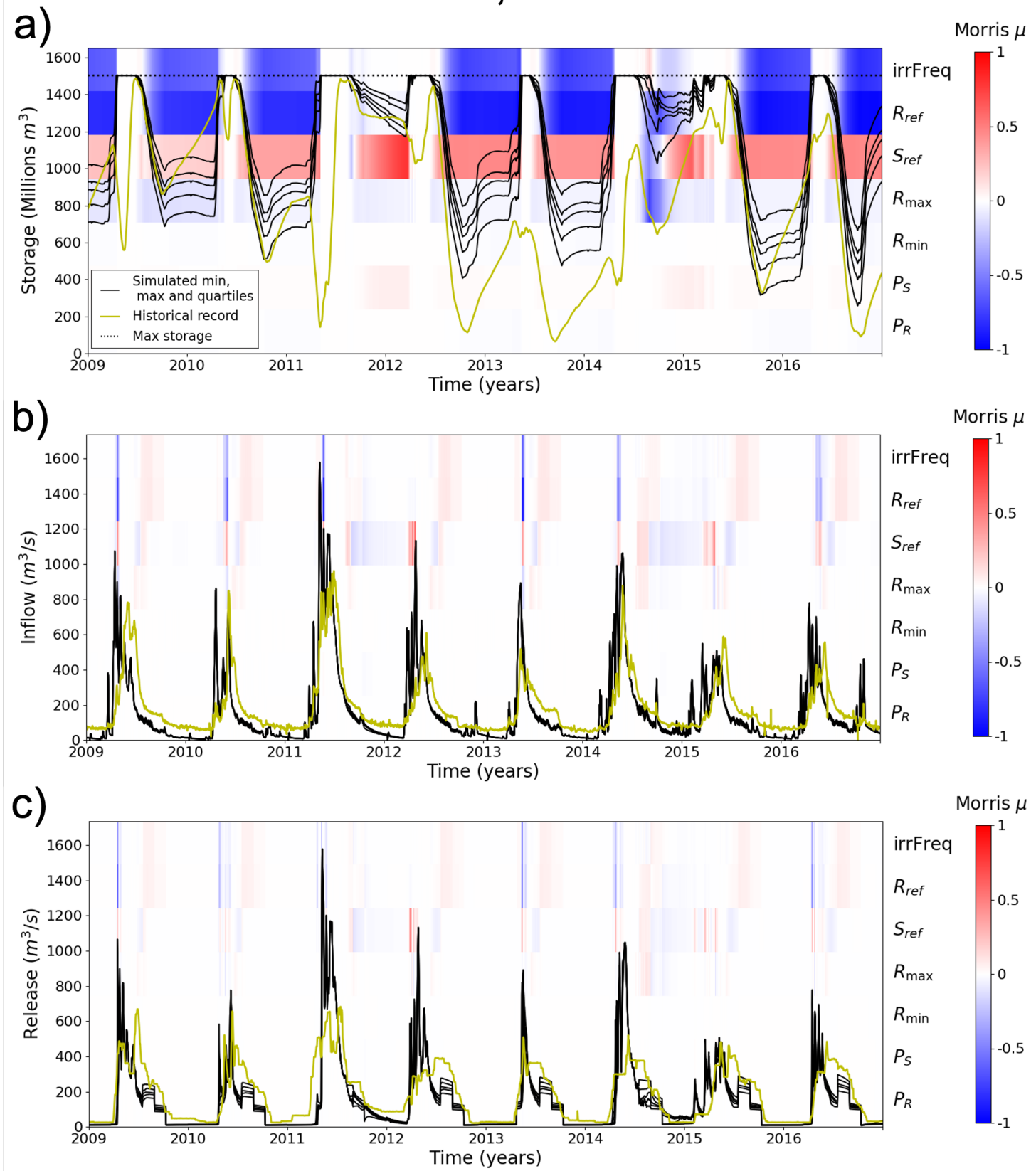

Figure S4. Simulated values ( $\max , \min$ and quartiles, shown with black lines) with historical values (gold line), and sensitivity to input variables (background), for (top to bottom): Palisades reservoir's inflow, release and storage. 

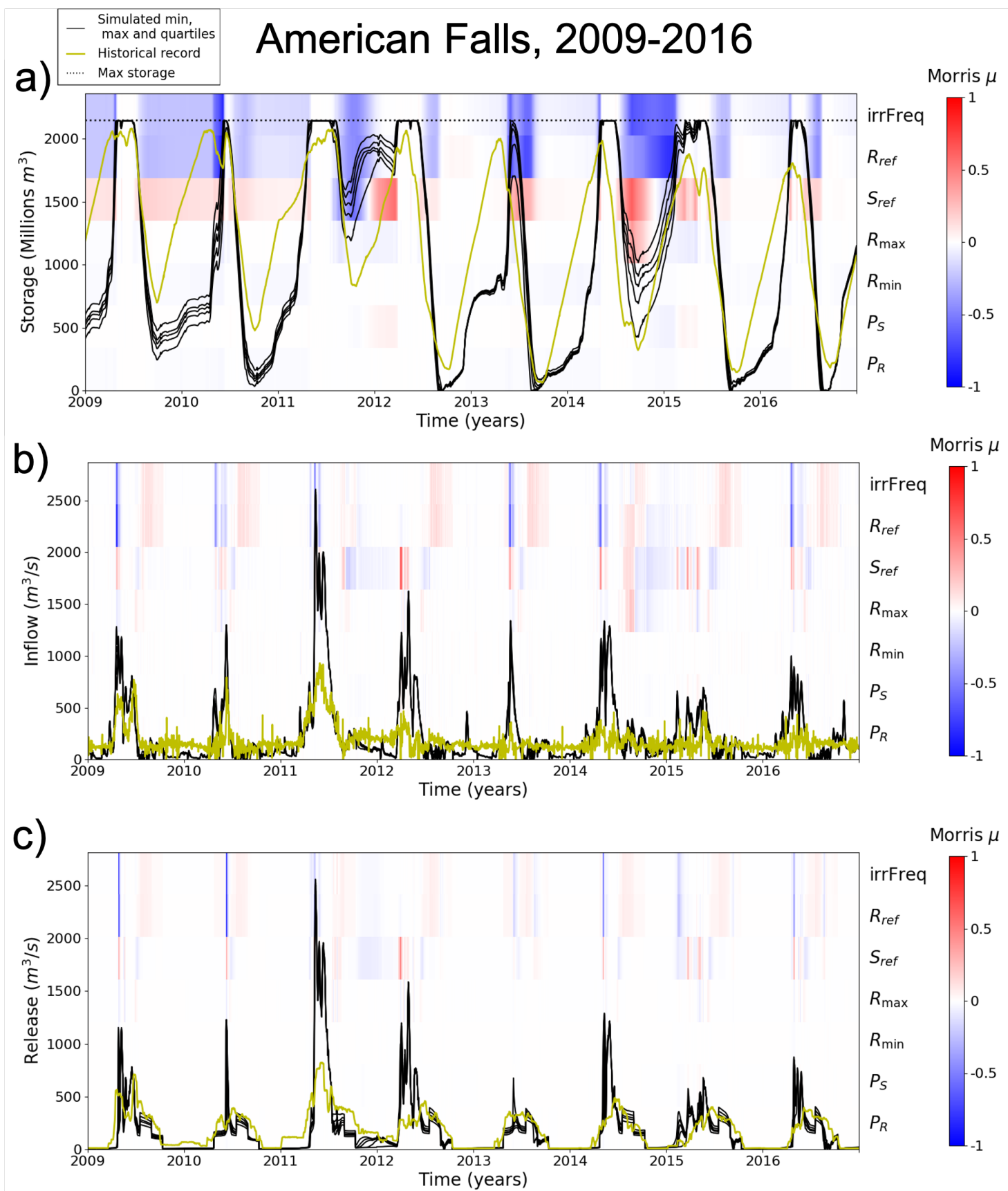

Figure S5. Simulated values (max, min and quartiles, shown with black lines) with historical values (gold line), and sensitivity to input variables (background), for (top to bottom): American Falls reservoir's inflow, release and storage. 

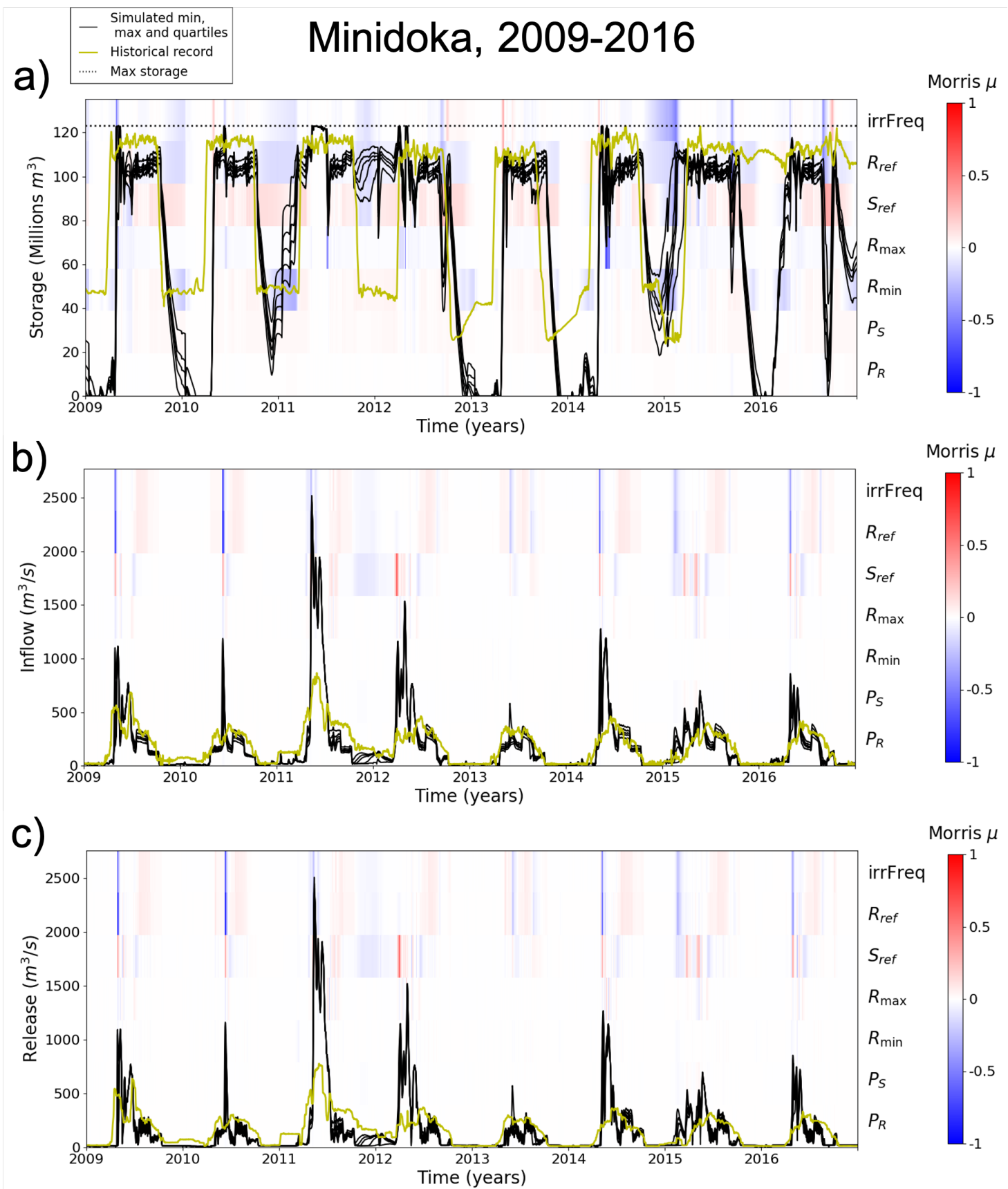

Figure S6. Simulated values (max, min and quartiles, shown with black lines) with historical values (gold line), and sensitivity to input variables (background), for (top to bottom): Minidoka reservoir's inflow, release and storage. 

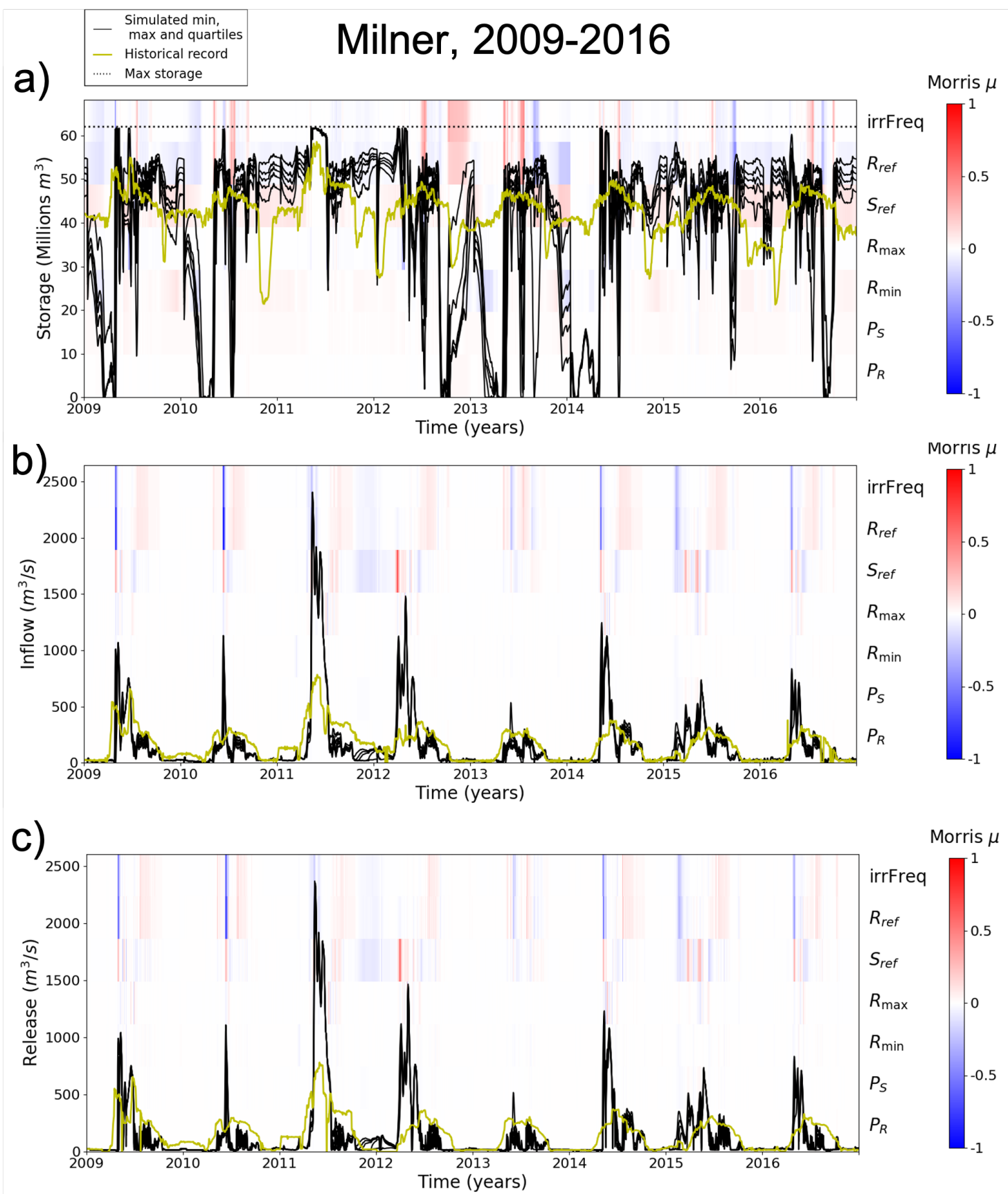

Figure S7. Simulated values ( $\max , \min$ and quartiles, shown with black lines) with historical values (gold line), and sensitivity to input variables (background), for (top to bottom): Milner reservoir's inflow, release and storage. 
Code and data availability. Core WBM code is available from the authors by request, and so is the code for this sensitivity analysis. Result data and code necessary to draw the figures are available online at https://github.com/charlesrouge/UpperSnakeRiver_reservoirs_WBM. 